\title{
Ordenación del Territorio y estrategias de planificación en los Caminos de Santiago Patrimonio Mundial
}

\author{
Regional Planning and Planning Strategies in the World Heritage \\ Camino de Santiago Pilgrim Routes
}

\author{
Xosé Somoza Medina' \\ Rubén C. Lois González² (1)
}

\section{Resumen}

El Camino de Santiago es hoy en día una ruta turística monumental de éxito internacional. La Organización de las Naciones Unidas para la Educación, la Ciencia y la Cultura [UNESCO] ha distinguido con la categoría de Patrimonio Mundial más de 2.500 kilómetros de itinerarios jacobeos en España, por los que cada año transitan más peregrinos, llegados de países vecinos y distantes, sean de mayoría católica o no. Las ocho comunidades autónomas por las que transitan estos Caminos presentan grandes diferencias en la estrategia de ordenación territorial que se deriva de los diferentes documentos aprobados, pasando de la conservación y protección de las sendas históricas en algunos casos a la promoción y posicionamiento del producto turístico en otros. En este artículo se explican los factores principales que explican el éxito del Camino y las estrategias de planificación de las diferentes comunidades autónomas.

Palabras clave: Camino de Santiago; delimitación; protección ambiental; turismo cultural; ordenación del territorio.

\begin{abstract}
Nowadays the Camino de Santiago is a monumental tourist route of international success. The United Nations Organization for Education, Science and Culture [UNESCO] has distinguished it with the World Heritage category and there are more than 2,500 kilometres of itineraries in Spain, which pilgrims from neighbouring or distant countries, whether Catholic or not, travel on each year. There are significant differences in the territorial planning strategy in the eight autonomous communities through which these routes cross. This is a result of the different approved documents, the conservation and protection of the historical trials in some cases, and the promotion and positioning of the tourist product in others. This article discusses the main factors behind the success of the Camino and the planning strategies of the different autonomous communities.
\end{abstract}

Keywords Camino de Santiago; delimitation; environmental protection; cultural tourism; regional planning.

1 Departamento de Geografía y Geología. Universidad de León, España. somoza@unileon.es

2 Departamento de Xeografía. Universidade de Santiago de Compostela, España. rubencamilo.lois@usc.es 


\section{Introducción}

El Camino de Santiago es un itinerario de peregrinación cristiana que en las tres últimas décadas ha experimentado una importante revitalización. Una antigua ruta medieval es utilizada por hombres y mujeres contemporáneos, que quieren dirigirse caminando hacia esta ciudad histórica gallega, patrimonio de la humanidad, con motivaciones tan diversas como las que mueven a la compleja sociedad contemporánea a la que pertenecemos (Roseman, 2004; Lois González, 2013; Greenia, 2014). Para algunos el Camino sigue siendo esencialmente una ruta religiosa, pero para la mayoría las motivaciones espirituales, paisajísticas, patrimoniales o de simple gusto por retornar a la movilidad lenta constituyen el impulso fundamental para dirigirse a Santiago (Maddrell, 2013; Santos Solla y Pena, 2014). Como se ha subrayado en varios trabajos recientes, el desplazamiento a pie durante varias jornadas, enlaza con varios atributos de la cultura actual: la noción de que un ejercicio continuado es una práctica saludable, que permite relacionarse con otros (la idea de comunitas), contemplar el paisaje en toda su dimensión, a partir de un desplazamiento lento, y reencontrarse con uno mismo (Frey, 1998; Coleman y Eade, 2004; Lois González, Castro Fernández y Lopez, 2015)

Para que este proceso de recuperación, o invención contemporánea, del Camino tuviese lugar se han debido combinar una serie de factores de origen diverso, tanto institucionales como populares, que justifican este éxito de revitalización de una vieja ruta medieval. Los primeros intentos de recuperación se dieron en época franquista, también con esta doble lectura, pues por un lado el régimen utilizó la restauración de monumentos del Camino en su estrategia propagandística de reivindicar la memoria católica y conservadora de la España del momento (Castro Fernández, 2010), y por otro lado en 1948-1949 se publicó el monumental libro (en tres volúmenes), Las peregrinaciones a Santiago de Compostela de los estudiosos Vázquez de Parga, Lacarra y Uría (1948/49). Para realizarlo, los tres recorrieron caminando la ruta hacia Santiago en los duros años de la Guerra Civil vestidos a la antigua usanza. Este libro permitió rescatar desde la investigación académica del momento el itinerario medieval seguido hacia Santiago, que sería precisado e ilustrado con datos historiográficos por universitarios españoles y extranjeros del ámbito de las humanidades desde los años 1950 (Moralejo, 1993).

En un período inmediatamente posterior, entre los años santos de 1965 y 1976, se debe destacar la acción de una serie de entusiastas de la recuperación de la ruta, bien investigadores interesados en dar a conocer la memoria de peregrinación a Santiago (historiadores, filólogos, archiveros, etc.), bien religiosos animados por la idea de recorrer el Camino, o ambas cosas a la vez (Santos Solla y Lois González, 2011; López, 2012) que comenzaron a señalizar la vía y a narrar su experiencia en crónicas o artículos. Se crearon las primeras asociaciones de amigos de Santiago, que desde siempre han mantenido la idealización del concepto tradicional de peregrinación, creando un discurso con gran influencia popular (Barreiro, 2009; Collins Kreiner, 2010). Todo este movimiento societario fue rápidamente asumido por las administraciones públicas, empezando por el Gobierno español que vio en el Camino la posibilidad de justificar la histórica europeidad de España, en un momento de incorporación del país a las Comunidades Europeas. De hecho, la primera exposición realizada por el Ministerio de Cultura español coincidiendo con el Tratado de Adhesión a la Comunidad Económica Europea [CEE] en 1985, giró sobre el legado del Camino, que se convirtió dos años después en el primer Itinerario Cultural reconocido por el Consejo de Europa.

Las escasas decenas de peregrinos que realizaban la ruta en los años 1970 constituyen el núcleo inicial de peregrinos contemporáneos a Santiago, que animará la fundación de asociaciones de amigos del Camino, que ya en 1987 celebraron su primer congreso en la ciudad de Jaca. Los estudiosos de la ruta (en este período, sobre todo historiadores y filólogos medievales), algunos religiosos y simples amantes de la caminata (católicos o no), construyeron la primera red de promoción del Camino, defendido según la fórmula medieval de marchar a pie o a caballo (a la que se añadiría la bicicleta), al menos 100 kilómetros, alojándose en lugares modestos y confraternizando con otros peregrinos o con las poblaciones del lugar (Lois González, 2013). La red de asociaciones de amigos creadas se extiende en la actualidad por todo el mundo, y cuenta con varios miles de miembros regularmente conectados en Italia, Alemania, Holanda, Reino Unido, Estados Unidos, Polonia y Canadá, por citar algunos de los ejemplos más destacados.

En los años 1980 y 1990 las comunidades autónomas atravesadas por la ruta, construyeron parte de su identidad y estrategia turística a partir de la promoción del Camino y, especialmente, desde la incorporación de la ruta del Camino francés a la lista de Patrimonio Mundial de la UNESCO en 1993. Del mismo modo actúan las ciudades y municipios de la ruta, interesados en construir redes prestigiosas de 
turismo cultural y urbano que atraigan visitantes (Soria i Puig, 1991; Caucci, 1993; Santos Solla, 1999). Desde hace decenios, todas estas instituciones públicas compiten por restaurar monumentos, pavimentar y arbolar el Camino para facilitar la marcha a pie y construir albergues o diversas instalaciones dirigidas al peregrino, con el fin de mejorar la ruta supuestamente histórica, hacerla más cómoda y justificar estrategias de buen gobierno centrado en el territorio y la cultura (Lois González y Somoza Medina, 2003; Pichel Pichel, 2004).

La eclosión mundial del Camino se produce cuando sus valores espirituales y culturales se popularizan a escala global a través de una serie de hechos puntuales. Uno de los momentos estelares de este resurgir se debe a la campaña promocional realizada por la Xunta de Galicia para publicitar el año santo de 1993, entre otras cosas, nombrando embajador para el mundo al cantante Julio Iglesias, además de invertir grandes sumas en la recuperación de las infraestructuras camineras. Por otro lado, personajes tan mediáticos como Paulo Coelho (1987) o Shirley MacLane (2001) escribieron sendos libros sobre su experiencia de peregrinación, obras de escaso interés para los auténticos caminantes, pero que se vendieron muy bien y contribuyeron a construir la imagen internacional del Camino. Un poco más serio y con el mayor éxito de ventas de la literatura caminera de toda la historia (se comercializaron aproximadamente cinco millones de libros), la obra de H. Kerkeling (2006), un cómico-animador muy popular de la televisión alemana, convirtió al Camino en un referente popular inexcusable en este país central de Europa. Más rigurosos, profundos e interesantes son los trabajos de C. Nooteboom (1996) o la coreana K. Hyosun (2011), que tratan con precisión su experiencia de caminantes a Santiago, desde la posición de un escritor consagrado o de una periodista de masas, muy reconocida en Corea del Sur. De nuevo, el Camino, el hecho de caminar durante jornadas anima la producción de textos escritos que, bajo la forma de libro y cada vez más a partir de formatos digitales, impulsan la recuperación entusiasta y popular de la peregrinación en un contexto contemporáneo. La influencia de Paulo Coelho, H. Kerkeling o K. Hyosun sobre los mercados de caminantes brasileño, alemán y coreano son evidentes y comienzan a ser estudiados (Torres-Feijó, 2012). Junto a la narración más o menos literaria, también la experiencia del Camino y la ciudad de Santiago han sido tratadas profusamente por el cine en distintas películas (Escudero Gómez, 2013), entre las que destacan: The Way (2010), realizada por E. Estévez y su padre Martin Sheen, un actor de origen gallego y de una localidad no muy alejada de Santiago; las españolas Tres en el Camino (2004), o Al Final del Camino (2009); Saint Jacques-Le Mecque (2006), la principal contribución francesa al género; Onde está a felicidade? (2011) del director brasileño Carlos Alberto Ricelli; o Ich Bin dann mal weg (2015), la versión cinematográfica de la obra de Kerkeling.

Para finalizar la explicación de los factores que justifican el éxito internacional del Camino un breve apunte sobre otros actores privados que animan la difusión de la ruta a Santiago. Los mismos son compañías turísticas y agencias de viaje de muy diferente tamaño y nacionalidad, a las que se han unido en los últimos años blogueros, mantenedores particulares de páginas web y grupos de antiguos peregrinos en un mundo que ha eclosionado en los últimos años, responsable de la publicación de millones de páginas web en internet y que debe de ser analizado en futuros trabajos. Por lo que respecta a las empresas de viaje, sus folletos promocionales han sido estudiados en la Tesis Doctoral de D. Santomil (2011), quien demuestra que sobre la idea común de caminar cada mercado nacional refuerza un atributo, acorde con sus preferencias. De hecho, países tan significativos en lo jacobeo como Alemania, Italia o Estados Unidos refuerzan el mensaje de peregrinación con una idea de naturaleza, paisaje y ecología en el primer caso (en los folletos y webs alemanas domina el verde en la presentación del Camino), de lugar religioso y de culto en Italia (los tonos rojos o encarnados adquieren más protagonismo en la rotulación), y de vuelta a la vieja Europa, al espíritu voluntario y solidario de la ruta en Estados Unidos, un país junto con el Reino Unido que ha generado la institución de los hospitaleros, personas que atienden desinteresadamente albergues varias semanas o meses al año, y que suelen tener preparación como fisioterapeutas para atender lesiones de los pies o musculares provocadas al caminar.

En resumen, una suma de factores diversos permite explicar el interés creciente de esta ruta histórica como destino turístico, ahora bien, cómo gestionan las administraciones públicas el Camino para mantener inalterables sus condiciones, a pesar de la cada vez mayor afluencia de peregrinos y de las transformaciones que provocan su éxito internacional. Cómo ha sido la evolución del planeamiento territorial ante un fenómeno tan extenso e importante que ha multiplicado el tránsito de peregrinos desde los 690 registrados en 1985 hasta los casi 280.000 de 2016. Este artículo trata de responder a estos interrogantes 
detallando los documentos y planes de ordenación territorial que sobre el Camino han aprobado comunidades autónomas y ayuntamientos en los últimos años.

\section{Fuentes y Metodología}

Todas las acciones desarrolladas en el Camino comentadas en el epígrafe anterior han generado un incremento continuo del número de peregrinos que se dirigen hacia Santiago, caminando al menos un centenar de kilómetros, o doscientos si es en bicicleta o a caballo (Figura 1). Esta distancia mínima fue establecida en su día por la iglesia compostelana en su intento de singularizar al peregrino tradicional y en los últimos años ha concitado un consenso total para definir el fenómeno (Herrero Pérez, 2008; Santos Solla y Lois González, 2011).

Con respecto al número de peregrinos su cifra no ha dejado de incrementarse desde el año 1970 cuando las fuentes oficiales contabilizan a 68 peregrinos, hasta 2016 año en el que se ha producido un nuevo record, 277.854 peregrinos, superando a los 272.703 registrados en el último año santo de 2010 (Oficina del Peregrino, 1970-2016). A este respecto, se debe señalar que los valores utilizados provienen de la Oficina del Peregrino gestionada por el cabildo compostelano, esto es, se trata de una fuente privada obtenida en un lugar donde los caminantes van a retirar un certificado de haber realizado la ruta (la denominada Compostela) (Xunta de Galicia y Universidade de Santiago, 2007-2010). En este registro existe una pequeña infravaloración del flujo (existen peregrinos no interesados en recoger el certificado) y un cierto sesgo religioso en las motivaciones consignadas. No obstante, con esta fuente se constata cómo en los años santos (cuando la festividad de Santiago coincide en domingo y se realizan grandes campañas de promoción de la ruta) existe un aumento considerable de caminantes. También se aprecia la dinámica seguida por la peregrinación a Santiago hacia convertirse en un producto global, pues desde 2012 los peregrinos españoles suponen menos del 50\% del total. Los países de procedencia, después de España (447\%), que más peregrinos contabilizan en 2016 son Italia, Alemania (ambas por encima de los 20.000 peregrinos), Estados Unidos (por encima de 15.000), Portugal, Francia (aproximadamente 10.000), Irlanda, Reino Unido (más de 6.000), Corea, Australia, Brasil y Canadá (por encima de 4.000) (Oficina del Peregrino).

Figura 1. Evolución del número de peregrinos a Santiago, que retiran la Compostela

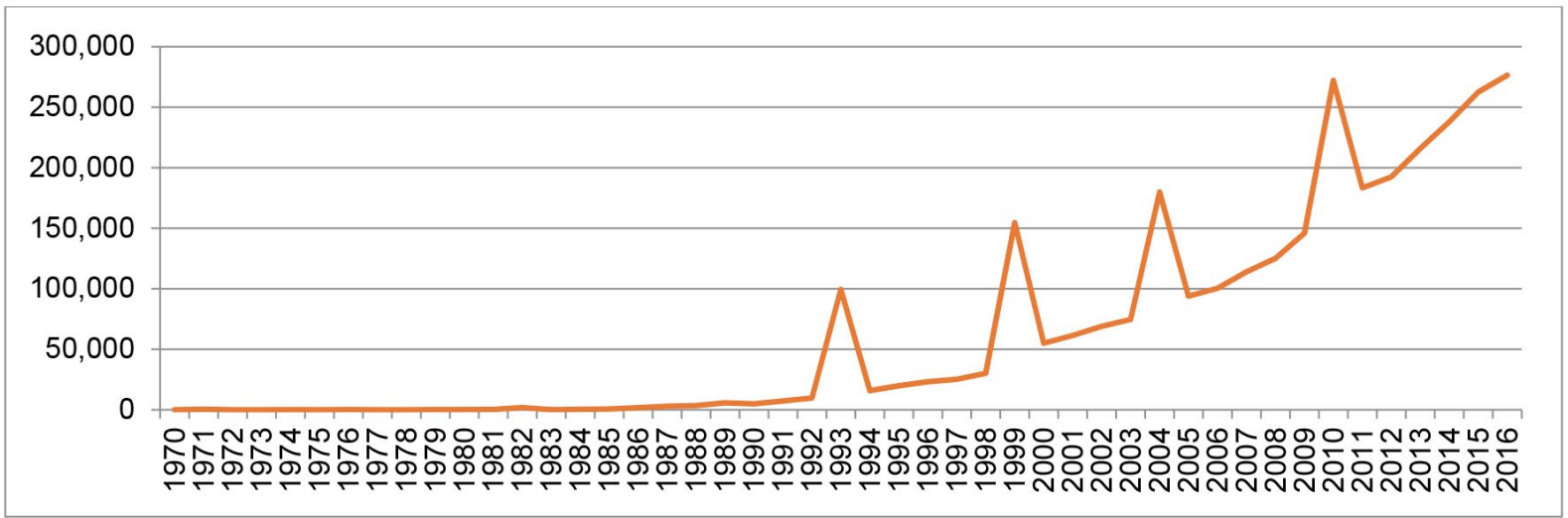

Fuente: Oficina del Peregrino. Elaboración propia.

El Camino de Santiago atraviesa espacios naturales, comunidades rurales y núcleos urbanos de diferente tamaño a lo largo de todo su recorrido. Es un eje histórico de comunicación que ha condicionado el poblamiento del territorio y que ha ido jalonando los distintos tramos con un abundante patrimonio arquitectónico.

Desde la década de 1970 se fueron aprobando diferentes instrumentos de planificación y proyectos de ordenación territorial que afectaban directamente al Camino, primero a las ciudades de mayor tamaño, a continuación a los núcleos rurales y finalmente a los espacios naturales. La ordenación y planificación era entonces sectorial y singular para cada tramo sobre el que se implementaba un determinado Plan, hasta que a finales de la década de 1990 comenzaron a aprobarse planes con un tratamiento integral del Camino. 
El Camino Francés fue considerado Conjunto Histórico Artístico en 1962 y desde entonces la legislación urbanística obligaba a realizar catálogos de patrimonio, con las correspondientes medidas de protección y conservación de los bienes inventariados a través de una normativa estricta (López Trigal, 1993). Los planes generales de ordenación urbana aprobados entonces establecieron la base para recuperar el patrimonio histórico artístico, que se desarrolló posteriormente con los Planes Especiales de Protección y Reforma Interior que fueron capaces de promover la rehabilitación residencial e impulsar la revitalización económica de estos núcleos históricos: con ejemplos en el Camino Francés en León (1993), Santo Domingo de la Calzada (1994), Mansilla de las Mulas (1995), Jaca (1996), Santiago de Compostela (1997), Melide (1999), Canfranc (1999), Pamplona (2001), Viana (2001), Ponferrada (2001), Puente la Reina (2005) y Villafranca del Bierzo (2016).

Las comunidades rurales que conforman buena parte de la realidad que atraviesa el peregrino no han tenido una figura de planeamiento exclusiva, pero sí se beneficiaron desde los años 1990 de la política europea de desarrollo rural. La iniciativa comunitaria "Liaison entre actions de développement de l'économie rurale" [LEADER] y el Programa Operativo de Desarrollo y Diversificación Económica de Zonas Rurales [PRODER], de ámbito nacional, en sus diferentes períodos, financiaron la actuación de los denominados Grupos de Acción Local, que tras un periodo inicial de análisis establecieron las líneas estratégicas a implementar para "reactivar" el territorio y la sociedad de sus ámbitos de actuación. Evidentemente, en aquellos espacios atravesados por el Camino, éste se convirtió en un eje aglutinador de intervenciones, con programas de actuación para acondicionar el sistema viario, recuperar el patrimonio arquitectónico y cultural local, generar nuevos equipamientos públicos, impulsar la creación de nuevas actividades económicas relacionadas con la hostelería y el turismo, o mejorar la transitabilidad del Camino con actuaciones concretas sobre el trazado, como aumentar el arbolado, cambiar el tipo de pavimento, crear nuevas áreas de descanso, mejorar el mobiliario urbano, o señalizar adecuadamente todas las vías.

Finalmente, la actuación planificadora sobre la naturaleza comienza con la catalogación del territorio a conservar a través de una determinada figura de protección, a la que le sigue la aprobación de un instrumento planificador que permite la utilización del espacio, el acondicionamiento de las sendas y la ordenación general del territorio bajo la perspectiva del desarrollo sostenible y el aprovechamiento turístico. Las posteriores legislaciones sobre el paisaje, a partir de la firma el año 2000 del Convenio Europeo en Florencia, indujeron a un tratamiento específico de este elemento, tan importante para el Camino, con normativas específicas en todos los futuros documentos de planificación.

La Ley estatal de Patrimonio Histórico de 1985 establecía la obligatoriedad de una delimitación precisa de los Bienes de Interés Cultural [BIC], que no existía para el Camino, ni siquiera tras su declaración como Patrimonio Mundial en 1993. Por ello la UNESCO al advertir sobre la dificultad de preservar una ruta de tan elevada extensión, recomendó a las autoridades españolas una delimitación precisa del bien declarado y un plan de actuación integral. Siguiendo estas determinaciones las comunidades autónomas realizaron estudios para detallar la extensión del Camino como paso previo para su planificación integral. Esta labor fue muy procelosa, por la necesidad de estudios previos, fases de exposición pública, recepción de alegaciones y nuevas propuestas de demarcación, de tal forma que las delimitaciones definitivas se fueron posponiendo desde la incoación inicial de los expedientes. Además, la delimitación establecía con claridad los límites del territorio afectado por el bien en una franja de 30 metros a ambos lados del Camino, en los que la protección ambiental impediría nuevos usos.

El presente artículo analiza de forma detallada la documentación oficial extraída de los procesos de delimitación y planificación integral que las administraciones públicas han aprobado sobre el Camino de Santiago, desde los primeros proyectos y decretos de principios de los años 1990 hasta el último plan estratégico de Asturias aprobado en noviembre de 2016.

\section{Resultados}

Los Caminos declarados Patrimonio Mundial (Figura 2) recorren más de 2.500 kilómetros a través de ocho comunidades autónomas y más de 300 municipios. A pesar de existir desde 1987 un Consejo de coordinación entre la Administración General del Estado y las administraciones autonómicas y un comité técnico para armonizar los criterios de planificación, lo cierto es que la ordenación en cada región presenta importantes diferencias, tanto en los instrumentos empleados, como en la escala utilizada o en la estrategia que define el tipo de intervención sobre el Camino. 
Figura 2. Mapa de los Caminos de Santiago declarados por la UNESCO patrimonio mundial

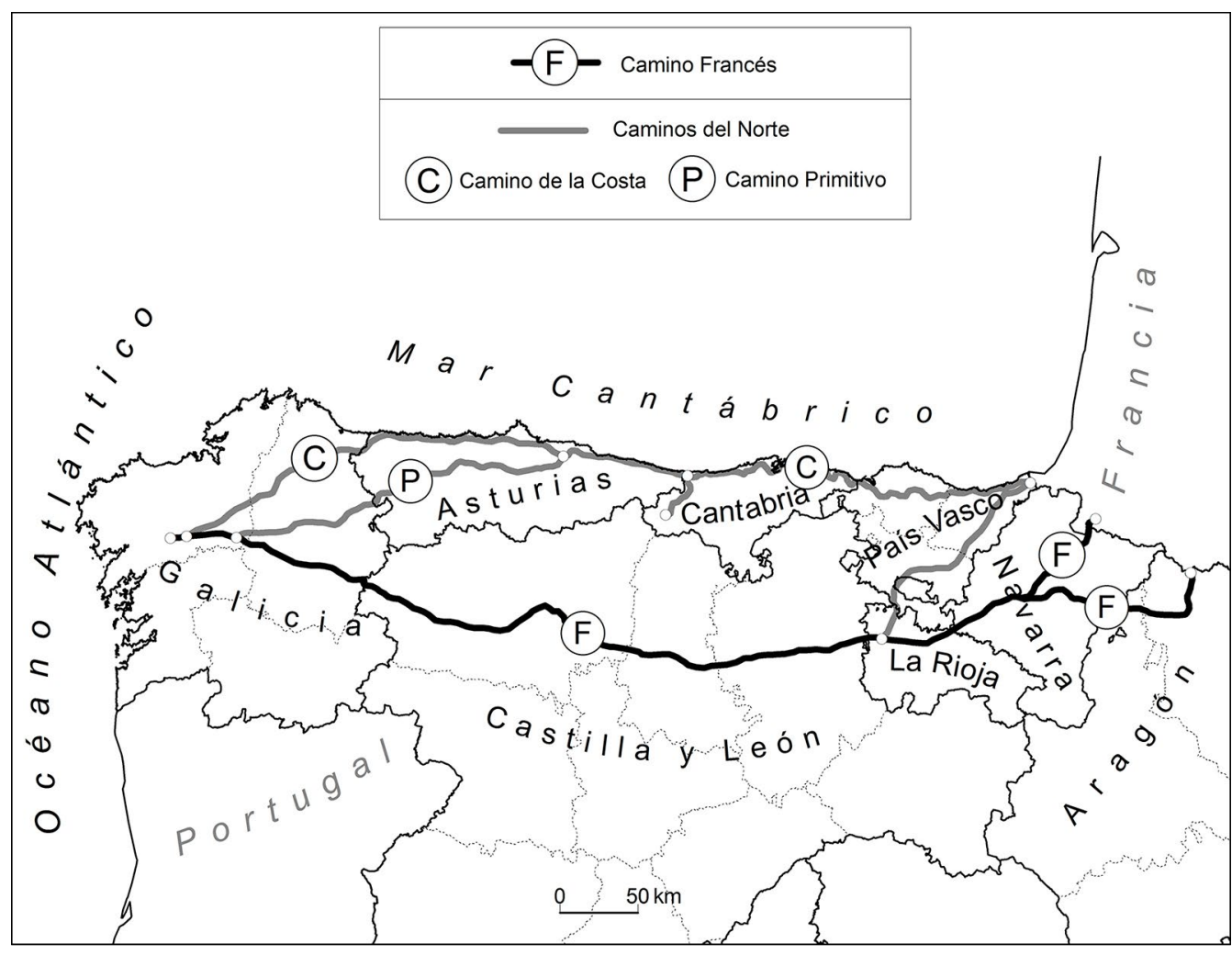

Elaboración propia.

\subsection{Aragón}

En el caso de Aragón el proceso de delimitación fue especialmente complejo, pues si bien la primera demarcación provisional se realizó en abril de 1993, siguiendo el trazado presentado a la UNESCO, en junio de 2001 este fue modificado con la intención de desplazar el itinerario y favorecer de ese modo la tramitación urbanística del recrecimiento del embalse de Yesa, en el conocido como ramal Sur, y de la construcción de la autovía A-21 de Pamplona a Jaca, en el ramal Norte, ambas actuaciones declaradas de interés general por el gobierno de España. La polémica creada obligó a una nueva rectificación en septiembre de 2002, pero las alegaciones de la Confederación Hidrográfica del Ebro y del Ministerio de Fomento, con sus correspondientes recursos ante el Tribunal Superior de Justicia de Aragón, impidieron la aprobación definitiva. En septiembre de 2015 se inauguró un nuevo tramo de la autovía, que tenía prevista su apertura completa en 2019, y en la nota de prensa facilitada por el Ministerio se exponía textualmente que entre las medidas de integración ambiental se destacaba la reposición del Camino de Santiago de manera paralela a la autovía y al norte de ella.

Antes de que esto ocurriera, en diciembre de 2007, se suscribió un Convenio de Colaboración entre el Ministerio de Fomento y la comunidad autónoma para la realización de un Plan de restauración, recuperación y acondicionamiento del Camino de Santiago, que incluía una extensa serie de actuaciones primando el denominado ramal Sur. La filosofía de este plan era la de concebir el Camino como un yacimiento arqueológico lineal, con sucesivas prospecciones que permitieron el afloramiento de múltiples vestigios de las calzadas antiguas (Figura 3). Se consolidaron muros perimetrales, nivelaron pendientes, compactaron terrenos, se protegió a los peregrinos con barandillas de madera en aquellos tramos de cierta peligrosidad y se levantaron puentes peatonales para salvar vaguadas y pequeños cauces. Se crearon además nuevas áreas de descanso con tejado, mesas y bancos, papeleras y fuentes y se actuó sobre la señalización del Camino con información de los diferentes núcleos y lugares de interés y con jalones indicativos en los cruces con referencia a la senda que se debía escoger y los kilómetros que faltaban hasta Santiago (Méndez de Juan, 2011). 
Figura 3. Restauración del Camino de Santiago en Aragón

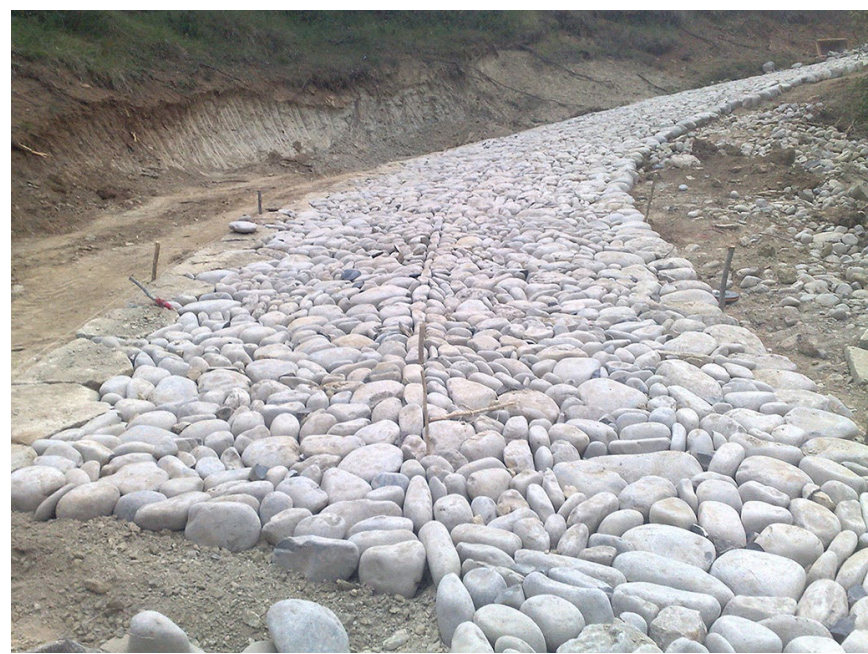

Fuente: Ministerio de Fomento, 2017

\subsection{Navarra}

Navarra aprobó la delimitación provisional del Camino en abril de 1988 y la definitiva en diciembre de ese mismo año. Sin embargo, al igual que en Aragón y tras sentencia del Tribunal Superior de Justicia de la comunidad foral, hubo de repetir el procedimiento, con aprobación inicial en abril de 1993 y definitiva en octubre de ese año. En el caso de Navarra parece no existir una preocupación especial por el Camino, pues hasta el momento no se ha tramitado ningún instrumento de planificación integral ni proyecto de restauración del Camino. De hecho, la propuesta inicial de ampliación de la categoría de Patrimonio Mundial a los Caminos del Norte aprobada por el Consejo del Patrimonio Histórico en 2011 incluía el Camino de Baztan, una ruta en Navarra de más de 110 kilómetros que enlaza la ciudad de Bayona con el Camino Francés en Pamplona a través del Puerto de Otsondo, pero finalmente en la redacción definitiva de la propuesta de ampliación se excluyó este itinerario.

\subsection{La Rioja}

La Rioja realizó la delimitación provisional en 1988 y la delimitación definitiva fue el soporte para el primer Plan Especial de Protección, Recuperación y Revitalización del Camino de Santiago, a su paso por esta región, aprobado en agosto de 1998. Este Plan Especial incluía además de las bandas de protección a ambos lados del Camino otras delimitaciones específicas, zonas de protección por riesgo de formación de núcleo, cerca de las localidades más pobladas, zonas de protección donde el Camino circulaba en paralelo a vías de comunicación, con una afección de $50 \mathrm{~m}$, y zonas de protección paisajística (Figura 4), para impedir la consolidación de actividades visual y ambientalmente agresivas en el entorno del Camino.

Figura 4. Ámbito de protección paisajística del Plan Especial del Camino de Santiago en La Rioja. (Escala original 1:50.000)

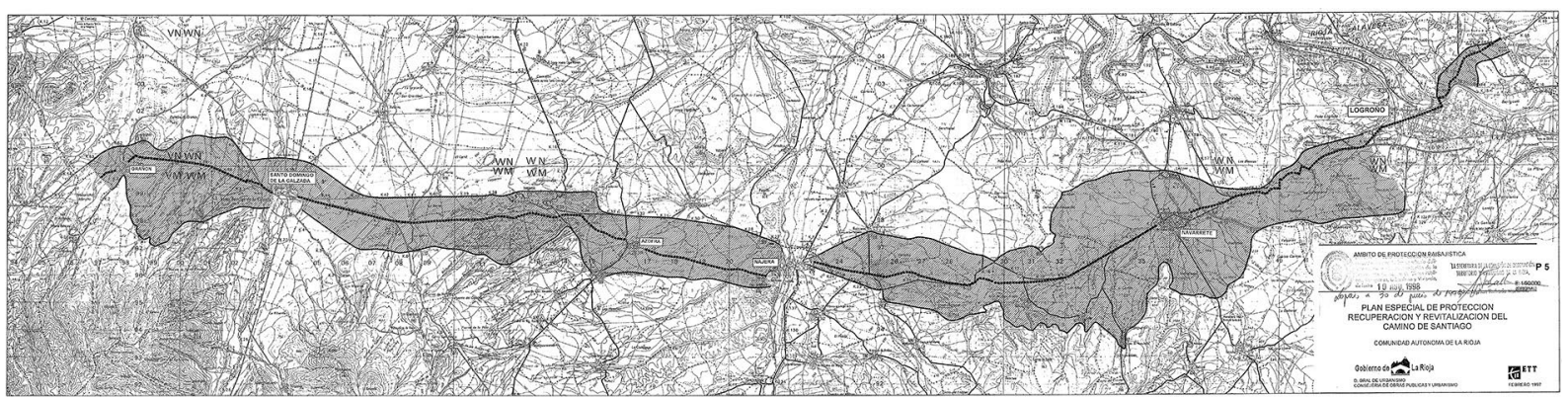

Fuente: Gobierno de La Rioja, 1998. 
En el Plan Especial se incluían también fichas específicas del catálogo de bienes singulares para 11 municipios y un plan de actuaciones (Figura 5) diseñadas con el criterio de garantizar una cómoda circulación de los viandantes, buscando la continuidad del itinerario peatonal, mejorando la ubicación y las características de las áreas de descanso, ampliando los recorridos monumentales de interés en los núcleos urbanos, reordenando las plazas públicas y buscando la integración de determinadas áreas de especial valor ambiental (Gobierno de La Rioja, 1998).

Figura 5. Programa de Actuación en el entorno rural del Plan Especial del Camino de Santiago en La Rioja. (Escala original 1:10.000)

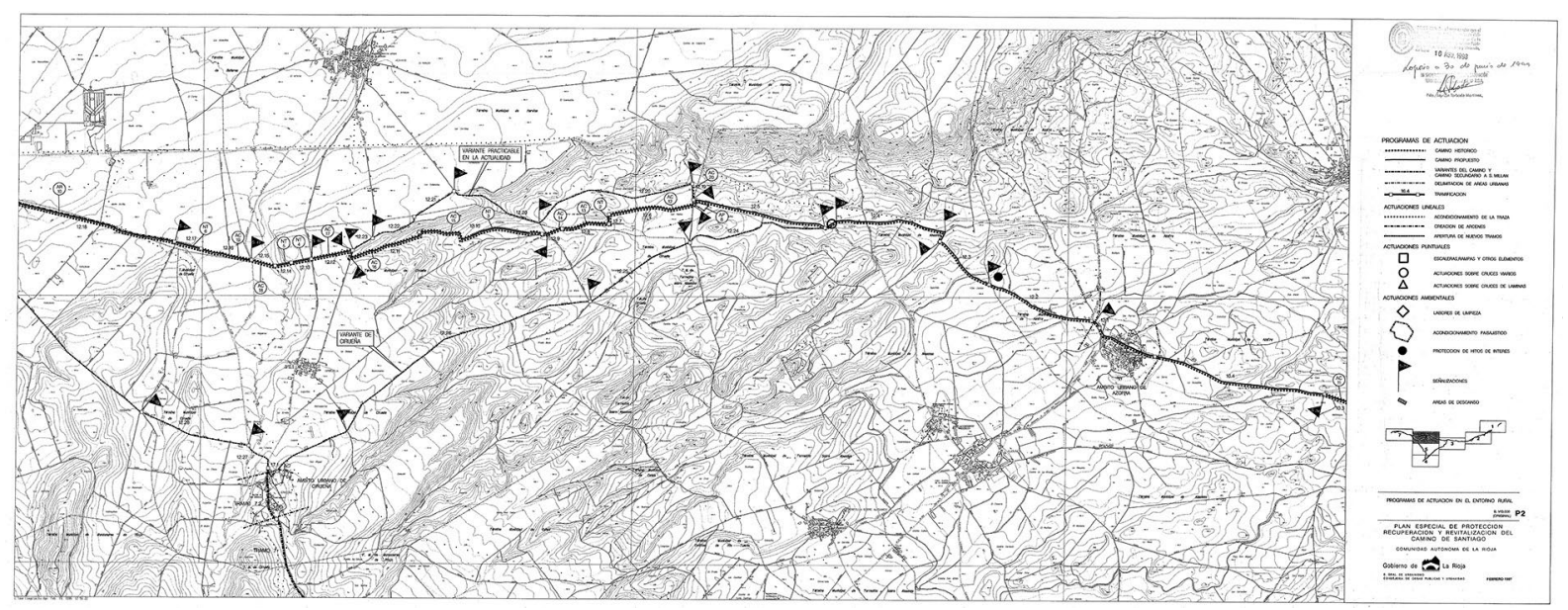

Fuente: Gobierno de La Rioja, 1998.

\subsection{País Vasco}

El País Vasco declaró los tramos del Camino del Norte en su territorio BIC y los delimitó en 2000, pero en un documento en el que más que detallar con precisión los tramos del Camino se enumeraban todos los hitos patrimoniales que podían visitar los peregrinos, más de 250 , y se incluía un relato de las poblaciones por las que debía pasar. El mapa del Camino, a escala 1:50.000 (Figura 6), carecía en este documento de cualquier tipo de referencia cartográfica que permitiera una correcta localización.

Figura 6. Delimitación del Camino de Santiago en el País Vasco según el Decreto 14/2000. (Escala original 1:50.000)

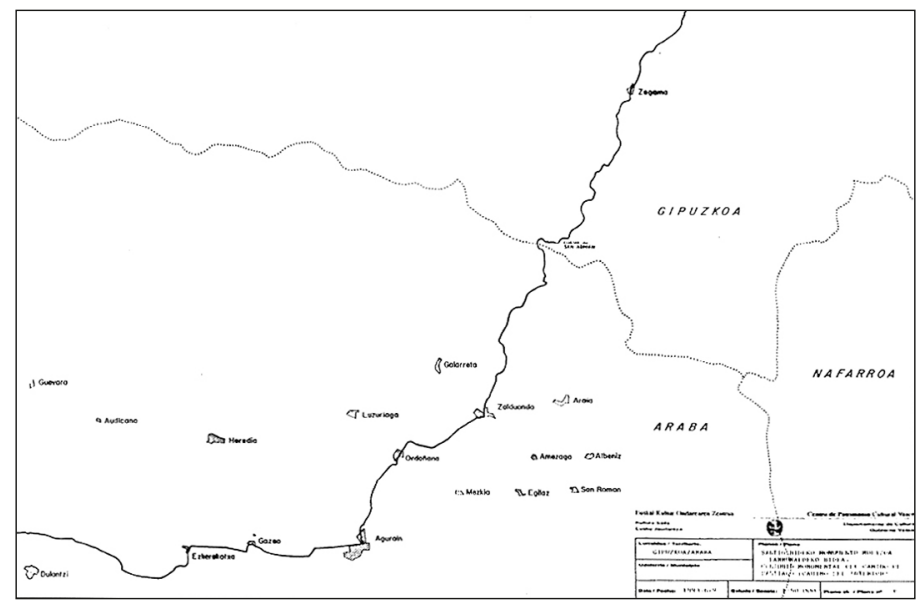

Fuente: Gobierno Vasco, 2000

Más tarde, en 2009, el gobierno vasco aprobó un Plan Director para el desarrollo y promoción cultural y turística de los Caminos de Santiago en este territorio, el de la costa y el interior que conecta con el Camino Francés, que citaba entre los objetivos estratégicos el de mejorar los servicios y equipamientos 
para mejorar el posicionamiento de Euskadi como destino jacobeo (Agencia Vasca de Turismo, 2009), introduciendo además una imagen promocional de marca distintiva (Figura 7).

Figura 7. Logo distintivo de los Caminos de Santiago en el País Vasco

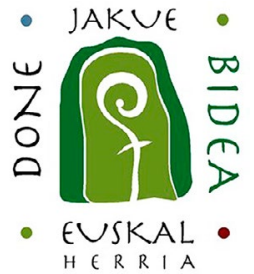

Fuente: Plan Director, 2009

En 2012 se aprobó una nueva delimitación del conjunto, con mapas a escala 1:20.000, en la que se exponía que en el anterior decreto se había favorecido más la unión de los hitos de significación jacobea que el propio trazado como camino físico, generando una indefinición que los estudios posteriores habían podido subsanar. Esos estudios se orientaron, según el texto del decreto 2/2012, a fijar una ruta real y física que fuera practicable, continua y segura a través de caminos históricos y calzadas públicas, evitando las carreteras, de tal manera que la delimitación establece un itinerario del siglo XXI, dinámico y vivo, desde el que se visualiza el patrimonio cultural de los lugares que atraviesa. Una definición, inserta en el texto legal, muy alejada de la visión de yacimiento arqueológico lineal del Plan de restauración aragonés.

\subsection{Cantabria}

Cantabria declaró en 2007 los tramos del Camino de Santiago en su territorio BIC, con la particularidad de definir dos itinerarios: la continuación del Camino de la Costa, con origen en Bayona y que recorre el País Vasco, Cantabria y Asturias y llega a Santiago atravesando la provincia gallega de Lugo; y por otro lado el denominado Camino Vadiniense o Ruta Lebaniega, que enlaza (o debería enlazar, ver figura 2) el Camino de la Costa con el Camino Francés a través del puerto leonés de San Glorio. La delimitación provisional se publicó en agosto de 2013 y la definitiva en octubre de 2015, tres meses después de su incorporación a la lista del patrimonio mundial de la UNESCO.

Figura 8. Delimitación del Camino de Santiago en Cantabria. (Escala original 1:25.000)

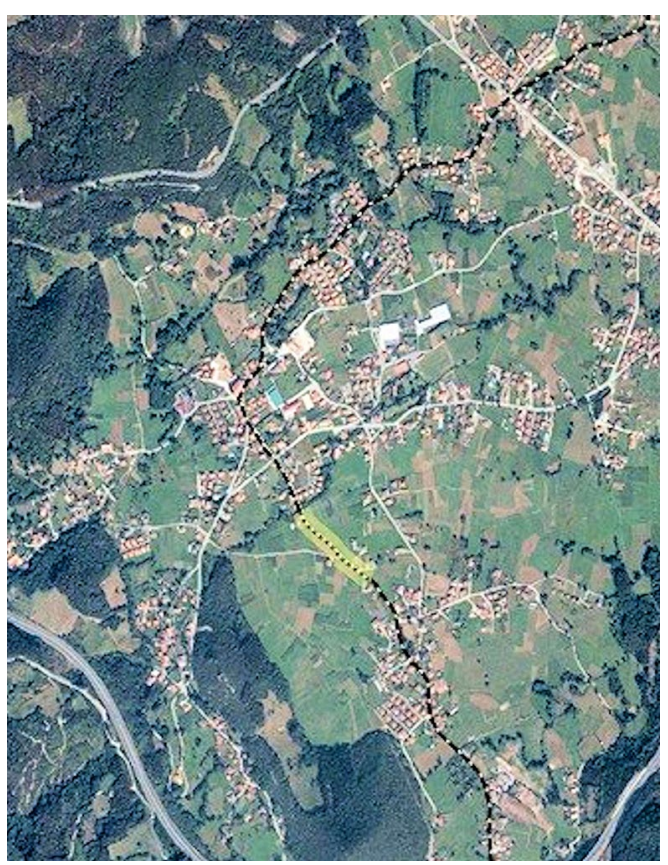

Fuente: Boletín Oficial de Cantabria 27/10/15. 
En el texto del Acuerdo del Consejo de Gobierno que delimita el Camino de la Costa se emplean siete páginas para relatar, como si se tratara de una bien documentada guía turística, las características de esta ruta jacobea y sólo tres líneas para definir el ámbito de protección, que a diferencia del resto de regiones establece en sólo 3 metros a ambos lados del eje del Camino en los ámbitos urbanos (30 metros en el espacio no urbanizable). Esta diferencia provoca que en amplios espacios de poblamiento disperso próximos a la costa cantábrica, de gran presión inmobiliaria, el Camino presente una protección exigua frente a nuevos desarrollos urbanísticos (Figura 8).

\subsection{Asturias}

A través del Decreto 63/2006 de 26 de junio la comunidad asturiana declaro Conjunto Histórico los Caminos de Santiago en su territorio y delimitó provisionalmente su trazado, incluyendo dos rutas y un itinerario de enlace entre ambas, rematando un proceso iniciado en 1994. Los tramos del Camino de la Costa atravesaban todo el territorio de Este a Oeste. Y lo que se denominaba Ruta Interior, agrupaba el tramo asturiano del Camino Primitivo de Oviedo a Santiago y la parte asturiana del Camino del Salvador, que unía la capital asturiana con el Camino Francés a través de la provincia de León entrando por el puerto de Pajares. En este caso el grado de protección "provisional" que se estipulaba era el de la banda de 30 metros en el espacio rural, mientras que en los ámbitos urbanos sólo quedaba afectada la parcela inmediatamente colindante a ambos lados de la vía. El carácter "provisional" se establecía hasta que los diferentes Planes Especiales que se deberían aprobar en cada Concejo no delimitaran definitivamente el Camino y sus ámbitos de protección.

A pesar de esta delimitación "extensa" de las rutas jacobeas asturianas, en la propuesta enviada por España a la UNESCO los Caminos de Santiago en Asturias se circunscribían únicamente a la ruta de la Costa y el Camino Primitivo. Uno de los problemas que estimó el comité técnico para su inclusión en la lista fue precisamente el carácter "provisional" de la protección de los Caminos asturianos.

En noviembre de 2016 el Principado de Asturias aprobó el Libro Blanco del Camino de Santiago en Asturias, en el que se reflejaba su estrategia planificadora con el horizonte del próximo año santo de 2021. En el primer capítulo de este Libro Blanco se expone con detalle el proceso de ampliación de la declaración de la UNESCO, resaltando como en la reunión del Consejo del Patrimonio Histórico Español celebrada en Burgos en marzo de 2011, algunas comunidades autónomas pretendieron incluir otras rutas en la propuesta, al estilo de la ampliación aprobada por la UNESCO en 1998 de los Caminos de Santiago en Francia. De hecho, la propuesta definitiva que se circunscribía a los Caminos del Norte, y no a los Caminos de Santiago en España como algunas regiones pretendían, obtuvo el rechazo en la votación de los representantes de Madrid, Valencia, Murcia, Ceuta y Castilla y León. La negativa de esta última comunidad autónoma a sumarse al proyecto de ampliación tuvo como consecuencia, según este Libro Blanco, que Asturias no pudiera incluir el Camino del Salvador en la propuesta, pues esta vía de enlace entre las ciudades de Oviedo y León no tendría continuidad en el tramo castellano y leonés. Lo mismo podría argumentarse en el caso del Camino Lebaniego cántabro, que sin embargo si fue aprobado por la UNESCO, dando como resultado un Camino de Santiago que no lleva a Santiago.

El Libro Blanco propone 10 líneas de actuación y 80 medidas entre las que figura la elaboración de un Plan supramunicipal de protección del Camino y el fomento del estudio de nuevas rutas, sin que ello supongo la ampliación de los Caminos declarados BIC, para no "extender ilimitadamente la protección..., configurándose como rutas turísticas sin protección patrimonial” (2016, p. 133). Entre las medidas de actuación son bastante más numerosas las relacionadas con la promoción turística que las relativas a la conservación patrimonial.

\subsection{Castilla y León}

Castilla y León inició el expediente de delimitación del Camino Francés en 1993 y lo concluyó en 1999. En virtud del Decreto 324/1999 de 23 de diciembre, la delimitación del BIC quedó marcada por un ámbito de protección de 100 metros a ambos lados del eje del Camino. Diez años antes, en 1989, habían comenzado los trabajos para elaborar un Plan Especial de Protección para esta ruta que no llegó a ser aprobado (López Trigal, 1993), aunque parte de los trabajos fueron continuados por la Diputación de León que pretendía entonces aprobar un Plan Especial provincial. Desde el año 2000 la Consejería de Fomento contrató la realización de este Plan a una consultora, inicialmente limitada a las provincias de Palencia y Burgos, por el avance de los trabajos que se estaban realizando en León, aunque desde 2005 
se decidió unificar los documentos. En 2010 se aprobó provisionalmente el Plan Regional del Camino de Santiago (Figura 9), que incluía además de los 422 kilómetros del trazado delimitado del Camino Francés, otros 218 kilómetros de tramos alternativos. El Plan incluía una serie concreta de medidas para conseguir la revitalización del ámbito territorial asociado al Camino, a través de tres escalas de intervención: territorial, intermedia y próxima y cinco planes estratégicos: Plan de adecuación integral del Camino de Santiago, Plan de Redes dotacionales y dinamización del Camino, Plan de calidad ambiental y del paisaje en el Camino, Plan de desarrollo energético en el Camino y Plan de adecuación de los instrumentos de planeamiento en los municipios atravesados por el Camino. El ámbito de protección intensiva en suelo no urbano se fijaba en 100 metros a ambos lados de la vía y el de protección ambiental a 1 kilómetro. En suelo urbano se fijaban dos tipos de afección, lineal y de conjunto, y en suelo urbanizable se establecían directrices concretas para la reserva de espacios adyacentes y la continuidad del Camino (Andrés y Masiá, 2011).

Figura 9. Plano de información del Plan Regional de Ámbito Territorial del Camino de Santiago en Castilla y León. (Escala original 1:10.000)

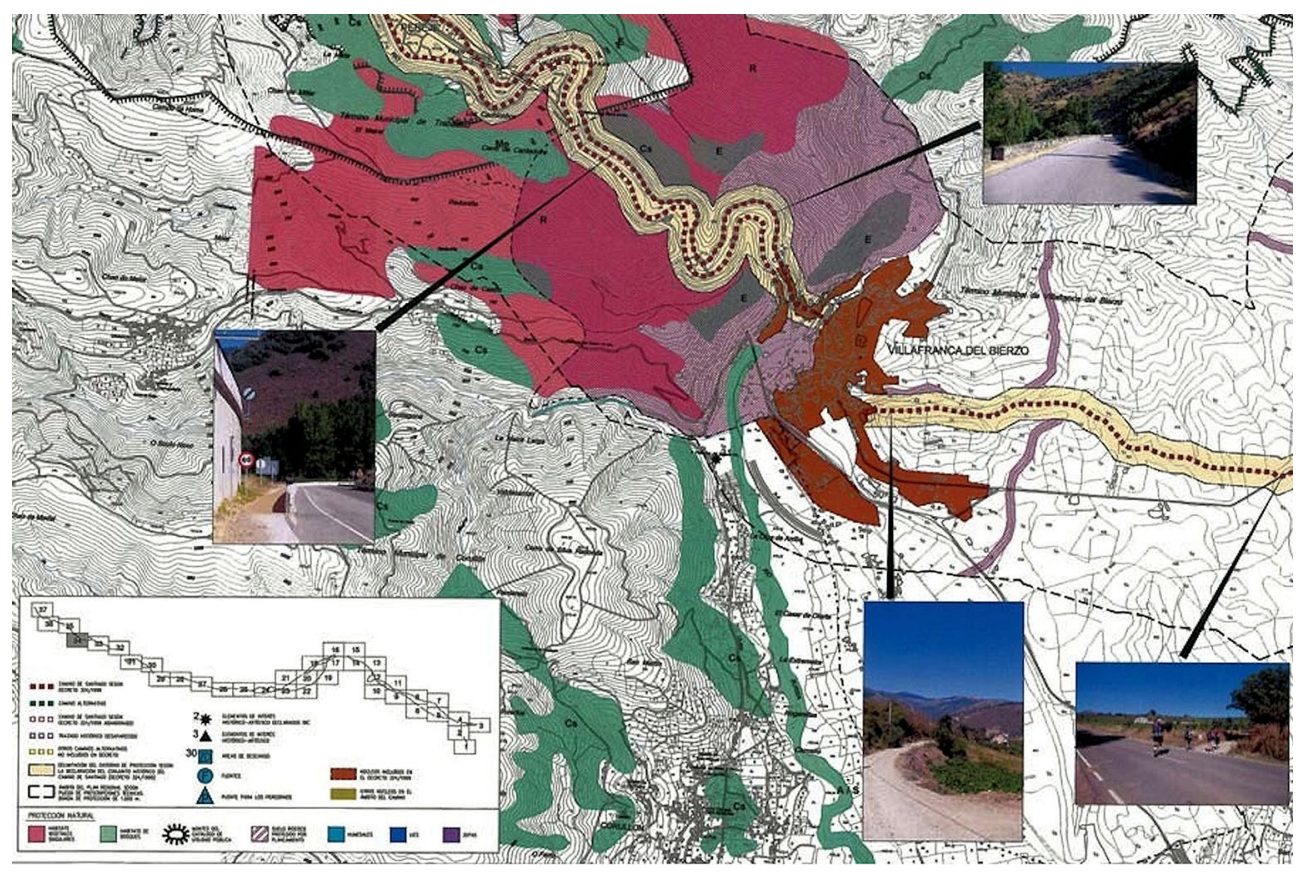

Fuente: Andrés y Masiá, 2011.

Sin embargo, el Plan Regional quedó estancado en el trámite final de la aprobación definitiva, con cruce de competencias entre la Consejería de Fomento y la de Cultura y Turismo que optaba por promover la Estrategia Regional de los Caminos a Santiago en Castilla y León. En la Estrategia se promovía la ampliación de la designación de BIC a todo el sistema de caminos jacobeos en la región, diferenciando entre el Camino Francés, los Caminos Históricos, los Caminos Tradicionales y los Caminos de Enlace o Conexión. En el mapa de la Figura 10 aparecen reflejados los 3.422 kilómetros de rutas jacobeas que contempla este documento, que en su metodología de actuación establece cuatro ejes estratégicos: coordinación y colaboración; protección de los valores; mejoras dotacionales; y difusión y promoción. Debemos recordar que la longitud de los Caminos distinguidos como Patrimonio Mundial por la UNESCO en toda España es de aproximadamente 2.500 kilómetros.

Una vez que el Plan Regional de Ámbito Territorial parece haberse frenado, lo que ha impulsado la Consejería de Fomento es la redacción de Planes Especiales de Protección en los ayuntamientos del Camino Francés. La Junta ha establecido como norma que todos los municipios de pequeño tamaño por los que discurre el Camino cuenten con un plan especial de este tipo para autorizar licencias de nueva construcción, siguiendo el ejemplo de la ciudad de Burgos, que cuenta con un Plan Especial de este tipo desde 1999. Desde que se puso en marcha esta estrategia de ordenación se han aprobado los Planes Especiales de Protección del Conjunto Histórico del Camino de Santiago a su paso por los municipios de Carrión de los Condes (2014), Valverde de la Virgen (2014), Camponaraya (2014), San Justo de la Vega (2015), 
Hontanas (2015), Sahagún (2015), Mansilla Mayor (2016), Molinaseca (2016), Itero de la Vega (2016), Villaturiel (2015), Vega de Valcarce (2016), Trabadelo (2016) y Villares de Órbigo (2017).

Figura 10. Mapa de la Estrategia Regional de los Caminos a Santiago. (Escala original 1:500.000)

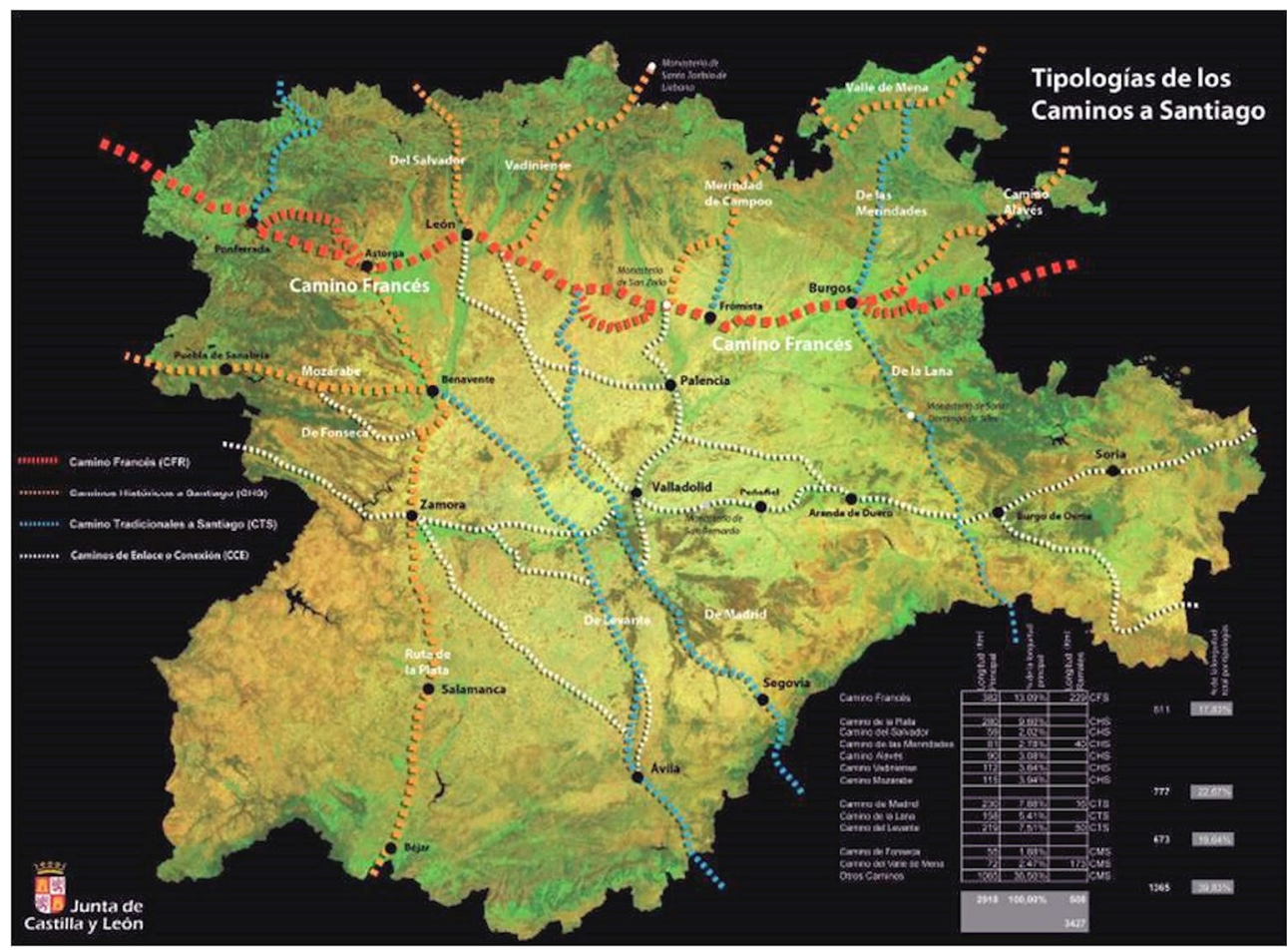

Fuente: Junta de Castilla y León.

Figura 11. Ordenación de La Faba en el Plan Especial de Protección del Conjunto Histórico del Camino de Santiago en Vega de Valcarce. (Escala original 1:1.000)

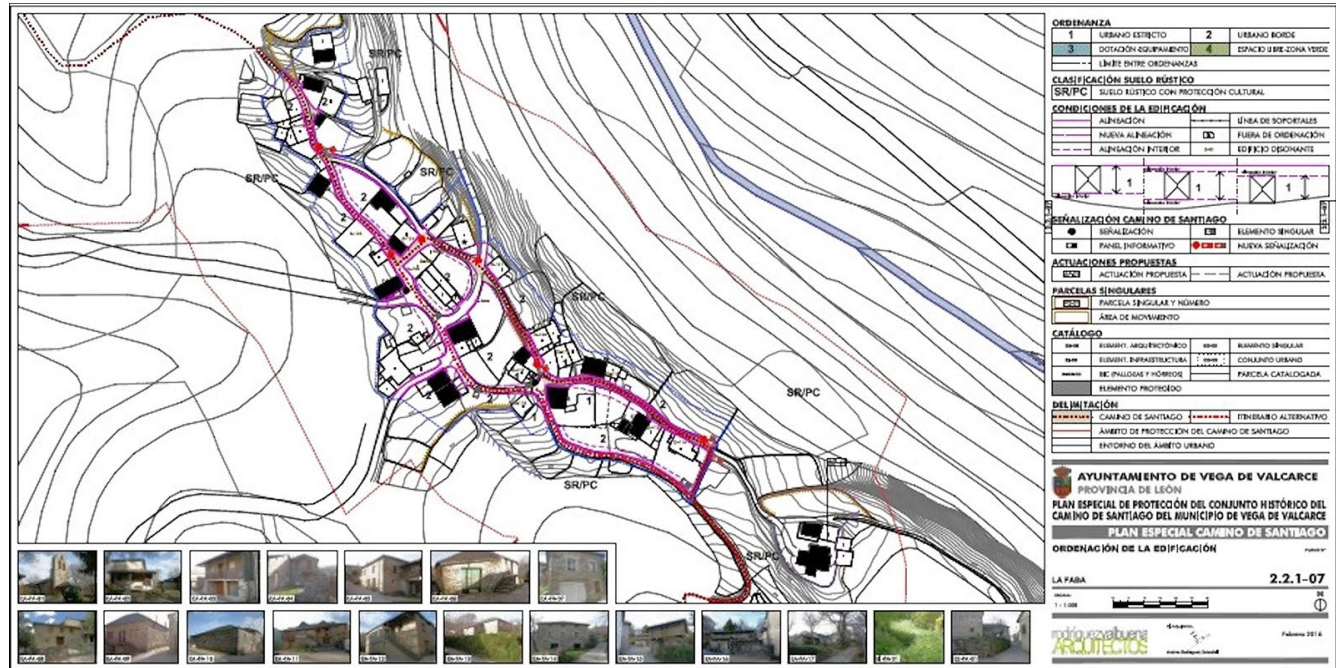

Fuente: Ayuntamiento de Vega de Valcarce, 2017.

\subsection{Galicia}

En Galicia, las normas subsidiarias de planeamiento provincial aprobadas en 1991 establecían un ámbito de protección de 100 metros a cada lado del Camino. Tiempo después, en 1996, la Ley de Protección 
de los Caminos fijaba al igual que en otras regiones las franjas de protección del Camino ( 3 metros) y del contorno (30 metros), además de un régimen sancionador y una normativa genérica mientras no se implementaba un Plan Especial regional que, según la disposición adicional de esta Ley, debía aprobarse en el plazo de dos años (Xunta de Galicia, 1996). En 2011 eran las Directrices de Ordenación del Territorio las que fijaban la necesidad de aprobar un Plan Especial para el Camino (Xunta de Galicia, 2011), y finalmente, la nueva Ley de Patrimonio Cultural de Galicia de 2016, establecía un nuevo plazo de dos años para aprobar un Plan Territorial Integrado de los Caminos de Santiago (Xunta de Galicia, 2016). En resumen, el tiempo ha ido pasando desde 1996 y a pesar de la obligatoriedad de desarrollar este Plan han transcurrido ya 20 años sin que se haya aprobado. Curiosamente, el mismo largo espacio temporal que media entre la delimitación provisional, en 1992, y la delimitación definitiva de los Caminos de Santiago en Galicia en 2012.

La realidad que justifica estos retrasos es compleja. Por un lado, los nuevos trabajos para la delimitación del Camino Francés llevados a cabo entre 2009 y 2012 descubrieron un gran número de variantes del trazado documentados y percibidos por la población como Camino de Santiago, lo que generaba serias dificultades para aplicar las diferentes franjas de protección y también para determinar los ámbitos susceptibles de recibir ayudas públicas para la rehabilitación de bienes privados. Por otro lado, el aumento en la afluencia de peregrinos era observado desde la Administración como un riesgo que debía ser estratégicamente tratado, diversificando las rutas y los Caminos de llegada a Santiago de Compostela para contrarrestar su posible saturación. Por este motivo, no sólo se promovieron otras rutas históricas, sino también nuevas ramificaciones de las ya existentes. La Ley de protección de 1996 ya oficializaba además del Camino Francés, los Caminos Portugués, Inglés, del Norte, de Fisterra y las rutas de la Plata y del mar de Ulla y Arousa, mientras que la nueva Ley de Patrimonio Cultural (Xunta de Galicia, 2016) incorporaba el Camino Primitivo y el Camino de Invierno, dejando abierta la posibilidad de catalogar nuevas rutas. Además, en esta Ley se mencionaba expresamente que los trazados de la ruta podían ser de tres tipos: principales, tramos históricos que permanecen en uso con características tradicionales; históricos, tramos históricos documentados que se perdieron física o funcionalmente; y funcionales, tramos alternativos de carácter cultural, ambiental o de seguridad para las personas usuarias (Figura 12). Por este motivo, los peregrinos que en 2016 avanzaban hacia Santiago de Compostela por el Camino Francés podían encontrarse en múltiples cruces con una doble señalización jacobea que ofrecía la posibilidad de seguir trazados complementarios, bien para recorrer sendas con vestigios históricos documentados o caminos para visitar lugares de interés, apartándose del más congestionado trazado tradicional.

Figura 12. Delimitación del Camino Francés en Melide, con indicación del trazado principal (negro), histórico (puntos en blanco) y complementario o funcional (puntos en negro) y zona de protección. (Escala original 1:20.000)

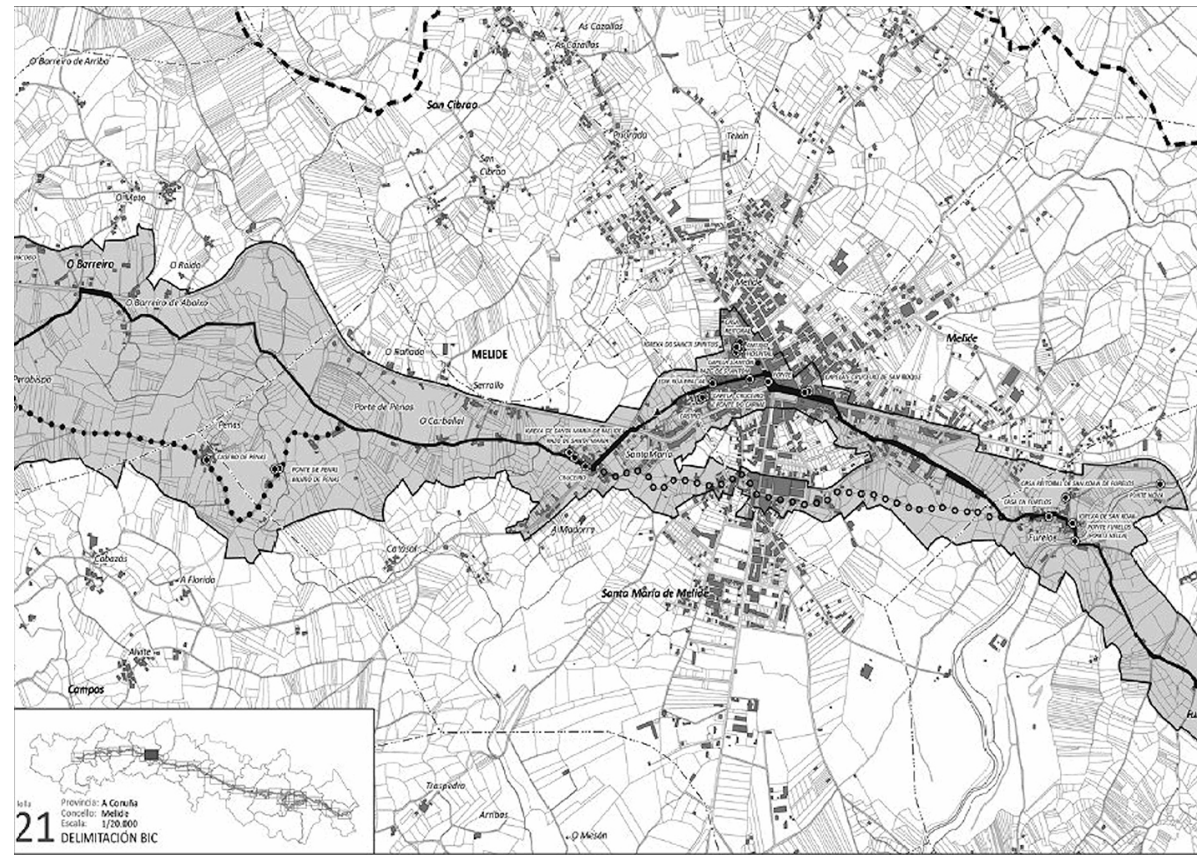

Fuente: Xunta de Galicia. 
Las comunidades autónomas son las que detentan la competencia en ordenación del territorio y planeamiento, y en el caso del Camino de Santiago, como queda establecido en cada normativa, son las respectivas Direcciones Generales de Patrimonio las encargadas de informar con carácter vinculante cualquier obra mayor o menor que se realice dentro del territorio afectado por el BIC. En realidad, éste es el mayor problema que percibe la población local, las dificultades que tienen para poder realizar cualquier cambio en sus propiedades por la tutela de "Patrimonio". Para tratar de solventar esta dificultad la Dirección General de Patrimonio Cultural de Galicia editó en 2012 una compleja Guía para la protección del Camino de Santiago, donde exponía las determinaciones y criterios que aplicaba para evaluar cualquier plan, programa o proyecto, y que todo técnico debía conocer si quería obtener un informe favorable y ver su trabajo realizado en el entorno del Camino (Xunta de Galicia, 2012).

\section{Discusión de Resultados}

Desde los años 1990, el Camino de Santiago alcanzó tal relevancia que surgieron los primeros ejemplos de tratamiento integral del Camino de Santiago con instrumentos específicos de planificación, tanto a escala regional como local. Una planificación del Camino que debía contar para su correcta implementación y pervivencia con el compromiso activo de la población local, la correcta coordinación de los diferentes gobiernos territoriales y la acumulación de sinergias entre el sector público y el privado.

Tabla 1. Estrategias de planificación territorial y económica

\begin{tabular}{|c|c|c|c|c|}
\hline $\begin{array}{l}\text { Comunidad } \\
\text { autónoma }\end{array}$ & Delimitación definitiva & $\begin{array}{c}\text { Planificación } \\
\text { Territorial }\end{array}$ & Planificación Económica & Estrategia \\
\hline Aragón & Pendiente Recursos & $\begin{array}{l}\text { Plan de restauración, } \\
\text { recuperación y } \\
\text { acondicionam. } 2007\end{array}$ & & Yacimiento arqueológico lineal \\
\hline Navarra & 1993 & & & $\begin{array}{c}\text { En contra de la declaración patrimonial } \\
\text { de nuevas rutas jacobeas (Baztan) }\end{array}$ \\
\hline La Rioja & 1998 & $\begin{array}{l}\text { Plan especial } \\
\text { de protección, } \\
\text { recuperación y } \\
\text { revitalización. } 1998\end{array}$ & & $\begin{array}{c}\text { Protección física y valorización } \\
\text { ambiental }\end{array}$ \\
\hline País Vasco & 2012 & & $\begin{array}{c}\text { Plan director para el } \\
\text { desarrollo y promoción } \\
\text { cultural y turística. } 2009\end{array}$ & $\begin{array}{l}\text { Mejorar el posicionamiento en el } \\
\text { mercado turístico. Itinerario del siglo } \\
\text { XXI, dinámico y vivo }\end{array}$ \\
\hline Cantabria & 2015 & & & $\begin{array}{l}\text { Delimitación patrimonial exigua. } \\
\text { Promoción turística }\end{array}$ \\
\hline Asturias & Sólo "provisional" & & Libro blanco. 2016 & $\begin{array}{c}\text { Creación de nuevas rutas turísticas sin } \\
\text { protección patrimonial }\end{array}$ \\
\hline $\begin{array}{l}\text { Castilla y } \\
\text { León }\end{array}$ & 1999 & $\begin{array}{l}\text { Planes especiales } \\
\text { de protección } \\
\text { municipales }\end{array}$ & Estrategia regional. 2009 & $\begin{array}{l}\text { Multiplicar los Caminos declarados } \\
\text { BIC en la región, mejorar señalización y } \\
\text { promoción turística. }\end{array}$ \\
\hline Galicia & $\begin{array}{l}\text { Camino Francés y } \\
\text { Camino Primitivo 2012, } \\
\text { Camino Costa } 2014\end{array}$ & $\begin{array}{c}\text { Guía para la } \\
\text { protección. } 2012\end{array}$ & $\begin{array}{l}\text { Ley de protección (1996) } \\
\text { y Ley de Patrimonio } \\
\text { Cultural (2016) }\end{array}$ & $\begin{array}{c}\text { Declarar todos los Caminos y } \\
\text { protegerlos patrimonialmente. } \\
\text { Diversificar las rutas. Trazado principal, } \\
\text { histórico y funcional. }\end{array}$ \\
\hline
\end{tabular}

Elaboración propia.

El análisis de las estrategias implementadas por cada comunidad autónoma (Tabla 1) refleja con total claridad la falta de coordinación entre ellas, y en algunos casos, entre diferentes departamentos de la misma administración regional. La planificación física-territorial camina por un lado y la estratégica-económica por otro.

La evolución positiva del número de peregrinos parece augurar su incremento moderado y sostenido durante los próximos años hasta 2021, cuando un nuevo año santo puede desbordar la ruta, y plantear graves problemas de saturación. El "éxito" de 2021 parece asegurado por la tendencia reciente del Camino, la pregunta es si a partir de 2022 la dinámica seguirá siendo la misma, teniendo además en cuenta el crisol de estrategias de planificación desplegado por las ocho comunidades autónomas analizadas.

\section{Conclusiones}

El Camino de Santiago es cada vez más una senda "diseñada" actual sobre una base física histórica. Una reinvención de un senderismo medieval con atributos contemporáneos y el confort y la seguridad 
que exige el peregrino del siglo XXI. Un fenómeno territorial complejo que ha necesitado de la acción, combinada o no, de diferentes instituciones públicas, personas individuales (académicas, famosas o anónimas) y asociaciones civiles de distinto tipo para alcanzar el éxito internacional.

La diferente visión, en principio contrapuesta, que fundamentan los planes de Aragón (yacimiento arqueológico lineal) y País Vasco (senda turística contemporánea), son una constante en todos los tramos de la ruta. Por ello el Camino es más que una peregrinación espiritual, más que historia de la cultura europea, más que turismo caminante y más que las tres cosas a la vez.

La preparación y promoción inicial de las pasadas décadas ha dado paso a una etapa actual de mejora de la transitabilidad a través del planeamiento y de promoción turística integral a través de los Planes Directores y Libros Blancos. Se ha conservado el patrimonio edificado, público y privado, el patrimonio natural y también el patrimonio cultural. Ahora es tiempo de mejorar la ruta en sí y la experiencia personal de cada peregrino.

No obstante, se ha comprobado cómo cada autonomía presenta unas estrategias diferentes para abordar tanto la protección y conservación del bien patrimonial como su promoción y difusión turística, desde la supuesta falta de interés que se presume por la total ausencia de documentos en Navarra a los conflictos que generan la profusión y solapamientos de planes y programas en Castilla y León. De la protección amplia y bien documentada de La Rioja a la exigua de Cantabria o provisional de Asturias. De la singularidad del Camino Francés en Navarra a la multiplicación de rutas jacobeas en Castilla y León o Galicia. De los estudios arqueológicos en Aragón al posicionamiento de las rutas vascas en el mercado jacobeo. La amenaza real del planeamiento regional es fracturar el Camino en segmentos que compitan entre sí, convirtiendo un producto único en ocho productos regionales que busquen su singularidad.

La planificación local es la escala básica para la mejora, tramo a tramo, del Camino, pero puede multiplicar la heterogeneidad del conjunto. Por eso es tan importante la labor supervisora de las autoridades regionales, aunque esta cuestión sea percibida negativamente por la población local.

El futuro del Camino es positivo siempre que las estrategias tomadas se implementen adecuadamente. Las rutas alternativas al Camino Francés van incrementando su afluencia y mejorando su red de albergues y servicios complementarios, impidiendo una saturación excesiva del trazado tradicional. La gran amenaza es precisamente esta, que el Camino pueda sucumbir por su propio éxito. La herramienta fundamental para impedir que esto ocurra es la planificación y la gestión territorial de un producto turístico-cultural complejo, de gran extensión y que necesita mantener la alianza perfecta entre la acción institucional, la promoción individual de los peregrinos y el sostenimiento activo de la población local.

\section{Referencias}

Agencia Vasca de Turismo. (2009). Plan Director para el desarrollo y promoción cultural y turística de los dos caminos de Santiago a su paso por Euskadi. Vitoria: Gobierno Vasco.

Andrés, C. y Masiá, Ll. (2011). Estrategias de planificación territorial. El Plan Regional del Camino en Castilla y León. Urban-e. Madrid: UPM. Recuperado de http://urban-e.aq.upm.es/pdf/Art\%C3\%ADculo\%20 PR\%20Camino\%20de\%20Santiago_def_1391943.pdf

Ayuntamiento de Vega de Valcarce. (2017). Plan Especial de Protección del Conjunto Histórico del Camino de Santiago a su paso por el municipio de Vega de Valcarce. Recuperado de http://www.vegadevalcarce. net/noti_amp.php?id=83

Barreiro, X.M. (2009). La Fundación de Occidente. El Camino de Santiago en Perspectiva Política. Madrid: Ed. Tecnos.

Castro Fernández, B. (2010). O redescubrimento do Camiño de Santiago por Francisco Pons Sorolla. Santiago de Compostela: Ed. Xunta de Galicia.

Caucci, P.(1993). L'Europa del pellegrinaggio. Milano: Jaca Book.

Coelho, P. (1987). El Peregrino. Barcelona: Planeta.

Coleman, S. \& Eade, J. (2004). Reframing Pilgrimage: Cultures in Motion. London: Routledge.

Collins Kreiner, N. (2010). The Geography of Pilgrimage and Tourism: Transformations and Implications for Applied Geography. Applied Geography, 20 (1), 153-164. https://doi.org/10.1016/j. apgeog.2009.02.001 
Escudero Gómez, L.A. (2013). La imagen urbana de Santiago de Compostela (España), un estudio de su representación pública, mediática, promocional y artística. Boletín de la A.G.E., 62, 265-294. Recuperado de http://www.age-geografia.es/ojs/index.php/bage/article/view/1578/1498

Frey, N. (1998) Pilgrim stories: On and off the road to Santiago. Berkeley, CA: University of California Press.

Gobierno de Cantabria. (2015). Resolución por la que se dispone la publicación del Acuerdo del Consejo de Gobierno mediante el que se procede a la definición del Camino de Santiago de la costa, a su paso por Cantabria y se delimita el entorno de protección del mismo. Boletín Oficial de Cantabria 27/10/15. Santander.

Gobierno de La Rioja. (1998). Plan Especial de Protección, Recuperación y Revitalización del Camino de Santiago a su paso por La Rioja. Logroño: Gobierno de La Rioja.

Gobierno del Principado de Asturias. (2016). Libro Blanco del Camino de Santiago del Principado de Asturias. Oviedo. Recuperado de https://www.asturias.es/Asturias/descargas/PDF_TEMAS/Cultura/ patrimonio/20161103_Libro_Blanco_Camino_Santiago_Principado.pdf

Gobierno Vasco. (2000). Decreto 14/2000, de 25 de enero, por el que se califica como Bien Cultural, con la categoría de Conjunto Monumental, el Camino de Santiago. Boletín Oficial del País Vasco 11/02/00. Vitoria.

Greenia, G. (2014). What is Pilgrimage?. In L. Harman (Ed.), A Sociology of Pilgrimage. Embodiment, Identity, Transformation. 8-28. London (Ontario): Ursus Press.

Herrero Pérez, N. (2008). Reaching Land's End: New Social Practices in the Pilgrimage to Santiago de Compostela. International Journal of Iberian Studies 21 (2), 131-149. https://doi.org/10.1386/ ijis.21.2.131_1

Hyosun, K. (2011). Camino de Santiago de Compostela: Camino Francés, Vía de la Plata, Camino Portugués. Kerkeling, H. (2006). Ich bin dann mal weg-Meine Reise auf dem Jakobsweg. Malik: Munich.

Lois González, R.C. (2013). The Camino de Santiago and its contemporary renewal: Pilgrims, tourists and territorial identities. Culture and Religion. An Interdisciplinary Journal. 14 (1), special Issue. Spaces of Renewal, A. Maddrell \& V. della Dora (Guest Eds.), 8-23. http://dx.doi.org/10.1080/14755610.20 $\underline{12.756406}$

Lois González, R.C., Castro Fernández, B. y Lopez, L. (2015). From Sacred Place to Monumental Space: Mobility Along the Way to St. James. Mobilities, 2015. http://dx.doi.org/10.1080/17450101.2015.10 $\underline{80528}$

Lois González, R.C. y Somoza Medina, J. (2003). Cultural tourism and urban management in northwestern Spain: The pilgrimage to Santiago de Compostela. Tourism Geographies, 5(4), 446-461. https://doi. org/10.1080/1461668032000129164

Lopez, L. (2012). La imagen de Santiago de Compostela y del Camino en Italia. Una aproximación desde la Geografía cultural. Santiago de Compostela: Tesis Doctoral, Departamento de Xeografía. Universidade de Santiago.

López Trigal, L. (1993). Políticas de rehabilitación das cidades e vilas camiñeras de León. En M ${ }^{a}$.P. TorresLuna, A. Pérez Alberti, y R. C. Lois-González (Eds.), Os Camiños de Santiago e o territorio. 793-805. Santiago de Compostela: Xunta de Galicia.

MacLane, S. (2001). The Camino: A Journey of the Spirit. Atria Books: Washington.

Maddrell, A. (2013). Moving and being moved: More-than-walking and talking on pulgrimage walks in the Manx lanscape. En Culture and Religion. An Interdisciplinary Journal. 14 (1) special Issue. Spaces of Renewal, A. Maddrell \& V. della Dora (Guest Eds.), 63-78. http://dx.doi.org/10.1080/14755610.2 $\underline{012.756409}$

Méndez de Juan, J.F. (2011). El tramo aragonés del Camino de Santiago (Camino Francés). En Urban-e. Madrid: UPM. Recuperado de http://urban-e.aq.upm.es/articulos/ver/el-tramo-aragone-s-del-caminode-santiago-camino-franc-s-/completo

Ministerio de Fomento. (2017). Restauración, Recuperación y Acondicionamiento del Camino de Santiago en Aragón. Proyectos de 1'5\% cultural. Recuperado de http://patrimoniohistorico.fomento. es/detalleProyecto.aspx? $\mathrm{e}=01477$ 
Moralejo, S. (Coord.) (1993). Santiago, Camino de Europa. Culto y cultura en la peregrinación a Compostela. Santiago de Compostela: Xunta de Galicia.

Nooteboom, C. (1996). El desvío a Santiago. Siruela: Madrid.

Oficina del Peregrino (1970-2016). Estadísticas de Peregrinación a Santiago de Compostela. Santiago de Compostela: Archicofradía del Apóstol.

Pichel Pichel, J.M. (2004). Arquitectura en el Camino, 1994-2004. Santiago de Compostela: Xunta de Galicia.

Roseman, S. (2004). Santiago de Compostela in the Year 2000: From Religious Center to European City of Culture. En Badone E. \& Roseman S. (Eds.), Intersecting Journeys: The Anthropology of Pilgrimage and Tourism (pp. 68-88). Retrieved from http://www.jstor.org/stable/10.5406/j.cttlxcq5w.7

Santomil, D. (2011). A imaxe exterior de Galicia no século XXI. Tesis Doctoral, Departamento de Xeografía. Santiago de Compostela: Universidade de Santiago de Compostela.

Santos Solla, X.M. (1999). Mitos y realidades del Xacobeo. Boletín de la A.G.E., 28, 103-119. Recuperado de https://dialnet.unirioja.es/descarga/articulo/1318654.pdf

Santos Solla, X.M. y Lois González, R.C. (2011). El Camino de Santiago en el nuevo contexto de los turismos. Estudios Turísticos, 189, 95-117.

Santos Solla, X.M. y Pena Cabrera, L. (2014). Management of Tourist Flows. The Cathedral of Santiago de Compostela. Pasos: Revista de turismo y patrimonio cultural, 12 (4). 719-735. https://doi.org/10.25145/j. pasos.2014.12.052

Soria y Puig, A. (1991). El Camino de Santiago: I. Vías, viajes y viajeros de antaño. Madrid: Ministerio de Obras Públicas y Transportes (MOPT).

Torres Feijó, E. (2012). Interesses culturais e âmbitos receptivos em dous romances sobre o Caminho de Santiago: "Frechas de ouro" e "O enigma de Compostela". Romance Notes, 52 (2), 135-150. https:// doi.org/10.1353/rmc.2012.0033

Vázquez de Parga, L., Lacarra, J.Mª. y Uría Ríu, J. (1948/49). Las peregrinaciones a Santiago de Compostela. 3 Tomos. Madrid: Consejo Superior de Investigaciones Científicas /CSIC).

Xunta de Galicia (1996). Lei de protección dos camiños de Santiago. Santiago de Compostela: Xunta de Galicia.

Xunta de Galicia (2011). Directrices de Ordenación Territorial. Santiago de Compostela: Xunta de Galicia.

Xunta de Galicia (2012). Guía para a protección do Camiño de Santiago. Santiago de Compostela: Xunta de Galicia.

Xunta de Galicia (2016). Lei de Patrimonio de Galicia. Santiago de Compostela: Xunta de Galicia.

Xunta de Galicia y Universidade de Santiago (2007-2010). Observatorio estatístico do Camiño de Santiago 2007, 2008, 2009 e 2010. Santiago de Compostela: Universidade de Santiago de Compostela-CETUR y Xunta de Galicia. 